\title{
Iterative solution of integral equations on a basis of positive energy Sturmian eigenfunctions.
}

\author{
George Rawitscher \\ Department of Physics, University of Connecticut, Storrs, CT 06269
}

\begin{abstract}
Years ago S. Weinberg suggested the "Quasi-Particle" method (Q-P) for iteratively solving an integral equation, based on an expansion in terms of sturmian functions that are eigenfunctions of the integral kernel. An improvement of this method is presented that does not require knowledge of such sturmian functions, but uses simpler auxiliary sturmian functions instead. This improved Q-P method solves the integral equation iterated to second order so as to accelerate the convergence of the iterations. Numerical examples are given for the solution of the Lippmann-Schwinger integral equation for the scattering of a particle from a potential with a repulsive core. An accuracy of $1: 10^{6}$ is achieved after 14 iterations, and $1: 10^{10}$ after 20 iterations. The calculations are carried out in configuration space for positive energies with an accuracy of $1: 10^{11}$ by using a spectral expansion method in terms of Chebyshev polynomials. The method can be extended to general integral kernels, and also to solving a Schrödinger equation with Coulomb or non-local potentials.
\end{abstract}




\section{INTRODUCTION}

Negative energy sturmian functions (S-F), introduced in the 60'ties by M. Rotenberg [1], found many useful applications, such as in the calculation of electron induced ionization collisions [2], [3], in the identification of resonances in nucleon-nucleus scattering [4], in the solution of a Schrödinger equation with non-local potentials [5], for a separable representation of scattering $t$-matrices [6] and in the solution of three-body Faddeev equations [7]. For the applications involving long range Coulomb forces, the analytical expressions for the Coulomb Green's functions, initially developed by L. Hosteler and R. Pratt [8] have found many applications [9]. sturmian functions form a complete, discrete set of eigensolutions of a Sturm-Liouville differential (or integral) equation, and form a complete set of basis functions. However the expansion of a wave function into a set of sturmian functions in many cases does not converge well [10], and methods to improve the convergence, such as Padé approximations, have frequently been utilized [11], [2]. The atom-atom potentials that occur in atomic physics calculation often have strong repulsive cores, in addition to a long range attractive part, and for such potentials it has been difficult to obtain a reliable set of sturmian functions. A method based on spectral expansions in terms of Chebyshev functions has been recently developed [12] that overcomes this difficulty, and hence the use of sturmian functions is again of interest. Nevertheless, for numerical calculations the expansions have to be truncated, and because of the slow convergence of sturmian expansions, an iterative method to correct for the truncation error becomes desirable. It is the purpose of this study to introduce such a method, based on the original Quasi-Particle method of Weinberg [13], and, for the purpose of testing it, apply it to the scattering of a particle from a potential as described by the Lippmann-Schwinger (L-S) integral equation. Since, the method described here should be applicable to more general kernels of an integral equation, the present study is done in anticipation of solving the more complicated two-dimensional integral equations that occur in the solution of three-body equations in configuration space [10], [14]. The expected accuracy is to be better than 6 to 7 significant figures, desirable for doing atomic physics calculations. By comparison, the solution of three-body equations for nuclear physics

applications, done commonly in momentum space [15], achieve an accuracy not better than four significant figures [16].

The present paper examines the convergence of several iteration methods for the solution 
of the L-S equation describing the scattering of a particle with positive energy $E$ by a local potential $V$ in one dimension. The insights gained from this example is to guide one for solving a more general integral equation. The method initially suggested by Weinberg [13], also called the "Quasi-Particle" method Q-P, requires sturmian functions that are the eigenstates for the exact integral operator. The present method, denoted as method $\mathcal{S}_{2}$, differs from the Q-P method in that it uses auxiliary sturmian functions that are based on an auxiliary potential $\bar{V}$ that is not the same as the potential $V$. Since it may be computationally easier to obtain the auxiliary sturmians rather than the sturmians for the original integral operator method $\mathcal{S}_{2}$ may prove advantageous for the case of very complicated integral kernels. For the $L-S$ example, the auxiliary sturmians are used to approximate the scattering potential $V(r)$ by a non-local separable representation $V_{N}\left(r, r^{\prime}\right)$ in terms of $N$ auxiliary sturmian functions and subsequently correcting for the truncation error $V_{R}=V-V_{N}$ by means of iterations. We find that the rate of convergence of the iterations does not depend significantly on the choice of $\bar{V}$, provided that the range of $\bar{V}$ is sufficiently larger than the range of $V$. The sturmian functions in all these cases are calculated in configuration space for a positive energy $E$, so that the asymptotic form of the approximated wave function is the same as the exact wave function. When solving for a bound-state wave function, it is customary the represent the potential by negative energy sturmians that are real, but for scattering cases, positive energy sturmians should be preferable. An advantage of the sturmian expansion method over the Fourier-Grid method [17] for positive energies is that the sturmian method emphasizes only the spatial region where the potential is non-negligible, the asymptotic part of the wave function being already incorporated into the sturmian basis, while in a Fourier-Grid method, the asymptotic part has to be obtained explicitly.

Since an accuracy of the scattering solution to $1: 10^{8}$ is envisaged, the sturmian functions are also required to have the same, or better, accuracy. Our approach for solving the SturmLiouville eigenvalue equation [12] utilizes a "spectral" expansion method into Chebyshev polynomials [18], [19], that permits one to prescribe a desired accuracy. With this method one can obtain sturmian eigenvalues and eigenfunctions with an accuracy of, for example, $1: 10^{11}$, and because of its stability, one can incorporate the effect of long-range tails of potentials, as was demonstrated in the calculation of the bound state eigenvalue of a HeliumHelium dimer [20]. The present calculations are performed in configuration space, and can be generalized to include asymptotic Coulomb functions. Contrary to what is often done at 
negative energies, our approach does not expand the Green's functions into a separable set of sturmians, but rather expands the potential $V(r)$ into such a set. The present method to calculate sturmian functions is similar to a method introduced previously [21], in that it does not use a square well potential sturmian basis set in terms of which the desired sturmians are expanded, and, being based on a spectral method, is considerably more precise. This additional feature permits a more accurate study of the iteration convergence properties.

In section II we present the formalism that defines the sturmian functions; section III describes the approximate Q-P methods $\mathcal{S}_{1}$ and $\mathcal{S}_{2}$ that extend Weinberg's Q-P original iterative method so that the use of sturmian functions for the integral kernel are avoided, and auxiliary sturmians are used instead; in section IV numerical examples are presented for the case that the integral kernel is of the Lippmann-Schwinger type, and section $\mathrm{V}$ contains the summary and conclusions. Appendices A, B, C and D contain further details.

\section{NOTATION AND EQUATIONS}

The one-dimensional Schrödinger equation for the $L=0$ radial wave function $\psi(r)$ is

$$
\mathcal{D} \psi=V \psi
$$

where

$$
\mathcal{D}=\frac{d^{2}}{d r^{2}}+E
$$

is the differential operator, $E$ is the energy assumed positive here, $V$ is the scattering potential. These quantities are in units of inverse length squared, and were transformed from their energy units by multiplication with the well known factor $2 \mu / \hbar^{2}$. The wave number $k$ is related to the energy by $E=k^{2}$. The corresponding Lippmann-Schwinger integral equation for $\psi$ is

$$
\psi(r)=F(r)+\int_{0}^{\infty} \mathcal{G}_{0}\left(r, r^{\prime}\right) V\left(r^{\prime}\right) \psi\left(r^{\prime}\right) d r^{\prime}
$$

The undistorted Green's function is given by

$$
\mathcal{G}_{0}\left(r, r^{\prime}\right)=-\frac{1}{k} F\left(r_{<}\right) \times H\left(r_{>}\right)
$$

where $\left(r, r^{\prime}\right)=\left(r_{<}, r_{>}\right)$if $r \leq r^{\prime}$ and $\left(r, r^{\prime}\right)=\left(r_{>}, r_{<}\right)$if $r \geq r^{\prime}$. In the present zero angular momentum case

$$
F(r)=\sin (k r) ; \quad H(r)=\cos (k r)+i \sin (k r)
$$


According to Eqs. (3) and (4) the asymptotic form of $\psi$ is

$$
\psi(r \rightarrow \infty)=F(r)+S H(r)
$$

with

$$
S=-\frac{1}{k} \int_{0}^{\infty} F\left(r^{\prime}\right) V\left(r^{\prime}\right) \psi\left(r^{\prime}\right) d r^{\prime}
$$

Near the origin $\psi(r \rightarrow 0)=0$, because both $F$ as well as the integral term in Eq. (3) go to zero.

The sturmian functions $\Phi_{s}(r), s=1,2, \ldots$ obey Eq. (1) with $V$ replaced by $\Lambda \bar{V}$

$$
\mathcal{D} \Phi_{s}=\Lambda_{s} \bar{V} \Phi_{s}
$$

where $\bar{V}(r)$ is the sturmian potential chosen conveniently, that need not be equal to the scattering potential $V(r)$, and the $\Lambda$ 's are the eigenvalues to be determined. The sturmian functions $\Phi_{s}$ obey the boundary conditions

$$
\Phi_{s}(r \rightarrow 0)=0 ; \quad \Phi_{s}(r \rightarrow \infty)=\mathfrak{k}_{s} H(r)
$$

where the constant $\mathfrak{k}_{s}$ is determined by the normalization of the sturmian function. The functions $\Phi_{s}$ obey the integral equation version of Eq. (8)

$$
\eta_{s} \Phi_{s}(r)=\int_{0}^{\infty} \mathcal{G}_{0}\left(r, r^{\prime}\right) \bar{V}\left(r^{\prime}\right) \Phi_{s}\left(r^{\prime}\right) d r^{\prime}, \quad s=1,2,3, \ldots
$$

with the Green's function given by Eq. (4) for positive energies. The $\eta$ 's are eigenvalues of the operator $\mathcal{G}_{0} \bar{V}$, they form an infinite set with point of convergence at 0 , and are related to the $\Lambda$ 's according to $\eta_{s}=1 / \Lambda_{s}$. With the choice (44) and (5) of the Green's function the sturmians $\Phi_{s}$ obey the boundary conditions (9), and

$$
\mathfrak{k}_{s}=-\frac{1}{k \eta_{s}} \int_{0}^{\infty} F(r) \bar{V}(r) \Phi_{s}(r) d r
$$

The $\Phi$ 's are not square integrable for positive energies or orthogonal to each other. However, they are orthogonal if one includes the potential $\bar{V}$ as a weight function

$$
<\Phi_{s} \mid \bar{V} \Phi_{s^{\prime}}>\equiv \int_{0}^{\infty} \Phi_{s}(r) \bar{V} \Phi_{s^{\prime}}(r) d r=0 \text { for } \eta_{s} \neq \eta_{s^{\prime}}
$$

In the bra-ket notation above, $<\Phi_{s} \mid$ is not the complex conjugate of $\Phi_{s}$, as is usually implied. The validity of (12) can be shown by replacing $\left.\Phi_{s^{\prime}}\right\rangle$ by $\left.\Lambda_{s^{\prime}} \mathcal{G}_{0} \bar{V} \Phi_{s^{\prime}}\right\rangle$ in the integral (12), and 
subsequently replacing $\left\langle\Phi_{s} \bar{V} \mathcal{G}_{0}\right.$ by $\eta_{s}\left\langle\Phi_{s}\right.$. The result is $\left.\left\langle\Phi_{s} \mid \bar{V} \Phi_{s^{\prime}}\right\rangle=\eta_{s} \Lambda_{s^{\prime}}<\Phi_{s} \mid \bar{V} \Phi_{s^{\prime}}\right\rangle$, and if $\eta_{s} \Lambda_{s^{\prime}} \neq 1$, the above identity becomes absurd unless $\left\langle\Phi_{s}\right| \bar{V} \Phi_{s^{\prime}}>=0$. Because of the completeness of the sturmian functions, one has the identity

$$
\delta\left(r-r^{\prime}\right)=\sum_{s=1}^{\infty} \Phi_{s}(r) \frac{1}{\left\langle\Phi_{s} \bar{V} \Phi_{s}\right\rangle} \Phi_{s}\left(r^{\prime}\right) \bar{V}\left(r^{\prime}\right)
$$

which can also be written as

$$
\delta\left(r-r^{\prime}\right)=\sum_{s=1}^{\infty} \bar{V}(r) \Phi_{s}(r) \frac{1}{\left\langle\Phi_{s} \bar{V} \Phi_{s}\right\rangle} \Phi_{s}\left(r^{\prime}\right),
$$

depending on whether the delta function applies to the left or to the right of the integrand in an integral.

One can understand intuitively the properties of the $\Phi$ 's as follows [21]. As the index $s$ increases, the corresponding values of $\Lambda_{s}$ increase, and hence the potential $\Lambda_{s} \bar{V}$ increases in magnitude. If $\bar{V}$ is real and attractive and the real part of $\Lambda_{s}$ is positive, then the real part of $\Lambda_{s} \bar{V}$ becomes more attractive, and the corresponding eigenfunction $\Phi_{s}$ becomes more oscillatory inside of the attractive region of the well. So, from one $s$ to the subsequent one $s+1$ the eigenfunction acquires one more node inside of the well. According to flux considerations the imaginary part of $\Lambda_{s} \bar{V}$ has to be positive, i.e., the well has to be emissive [21]. Near the origin the flux is $\simeq 0$ since $\Phi \simeq 0$ however asymptotically the outgoing form

of the wave function produces a positive outgoing flux. This outgoing flux is generated by an emissive imaginary potential, exactly the opposite of the case of an optical potential, that absorbs flux. These properties will be verified in the numerical section below.

\section{A. Numerical properties of sturmian functions.}

In the examples given below, the scattering potential $V$ is of the Morse type with a repulsive core near the origin $\left(V_{P}\right)$, and the sturmian potential $\bar{V}$ is either also of the Morse type with the repulsive core suppressed $\left(V_{S}\right)$, but of the same range as the scattering potential $V_{P}$, or it is a potential of the Woods-Saxon type $\left(V_{W S}\right)$ with a range larger than that of either $V_{P}$ or $V_{S}$. The Morse potentials are formed by the combination of two exponentials

$$
\bar{V}(r)=V_{M} \exp (-r / \alpha+\beta / \alpha) \times[\exp (-r / \alpha+\beta / \alpha)-2]
$$

with parameters given in Table I, and the Woods-Saxon potential is given by 


\begin{tabular}{|l||l|l|l|}
\hline & $V_{M}\left(\mathrm{fm}^{-2}\right)$ & $\alpha(\mathrm{fm})$ & $\beta(\mathrm{fm})$ \\
\hline \hline$V_{P}$ & 6 & $1 / 0.3$ & 4 \\
\hline$V_{S}$ & 6 & $1 / 0.3$ & 0 \\
\hline
\end{tabular}

TABLE I: Parameters for two Morse-type potentials $\mathrm{P}$ and S.

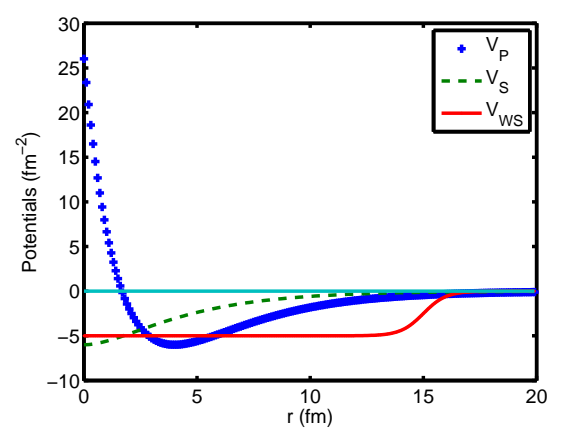

FIG. 1: Three sturmian potentials as a function of radial distance, given by Eqs. (15) and (16)).

$$
V_{W S}=V_{0} /\{1-\exp [(r-R) / a]\}
$$

with $V_{0}=-5 \mathrm{fm}^{-2}, R=15 \mathrm{fm}$ and $a=0.5 \mathrm{fm}$. The resulting dependence of these potentials on $r$ is illustrated in Fig. 1,

The spectrum of the eigenvalues $\Lambda_{s}$ for the two Morse-type potentials, with $k=0.5 \mathrm{fm}^{-1}$ is illustrated in Figs. 2 and 3. Since the potential $V_{S}$ is entirely attractive, the real parts of $\Lambda_{s}$ are positive, while the imaginary parts are negative, in accordance with the argument

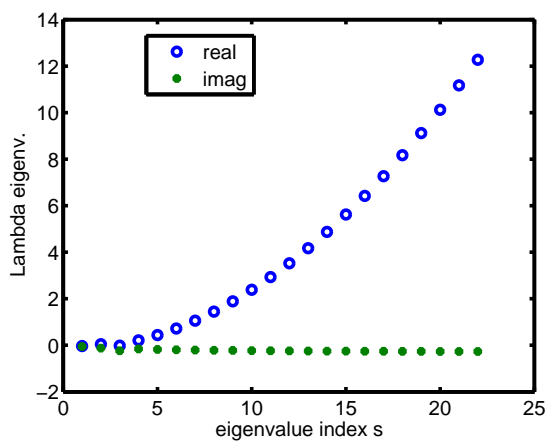

FIG. 2: The spectrum of the sturmian eigen values $\Lambda$ for the Morse Potential $V_{S}$ defined in Table I and illustrated in Fig. 1. The wave number is $k=0.5 \mathrm{fm}^{-1}$. 


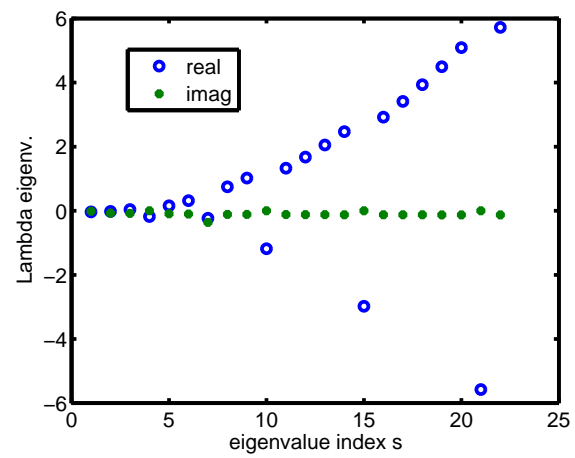

FIG. 3: Same as Fig. 2 for the potential $V_{P}$ defined in Table 1 . This potential has a repulsive core.

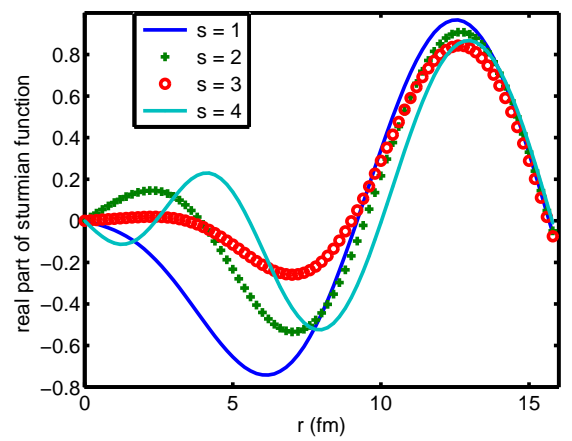

FIG. 4: Sturmian eigenfunctions for the potential $V_{S}$ defined in Table 1 for a wave number $k=$ $0.5 \mathrm{fm}^{-1}$. They are normalized such that asymptotically they all approach the outgoing Hankel function $H(r)=\cos (k r)+i \sin (k r)$.

given above. These sturmian eigenfunctions are obtained by an adaptation of Hartree's iterative method designed to obtain energy eigenvalues to the Schrödinger equation, and described in Appendix C. A list of these eigenvalues precise to 11 significant figures is given in the Appendix D. Some of the eigenfunctions are illustrated in Figs. 4 and 5, that show that at large distances where the potential becomes small, these functions become linearly dependent. The $\Phi_{s}$ used in the present examples are normalized such that asymptotically they equal the function $H(r)$, with unit coefficient.

Because potential $V_{P}$ has both a repulsive and an attractive part, the eigenvalues fall into two categories. In category I the eigenvalues $\Lambda$ have a positive real part and a negative imaginary part, and the corresponding eigenfunctions are large mainly in the attractive 


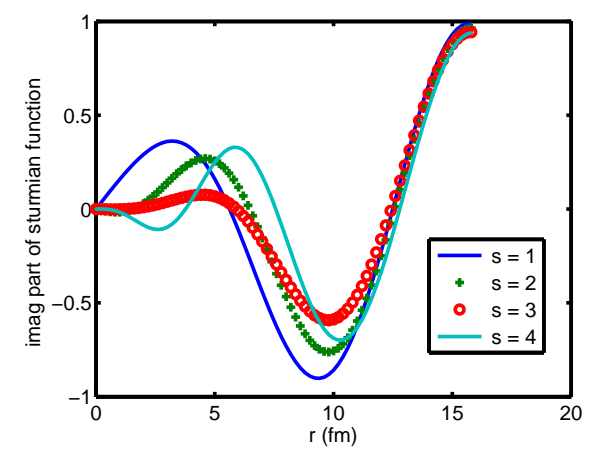

FIG. 5: Same as Fig 4 for the imaginary part of the sturmian functions.

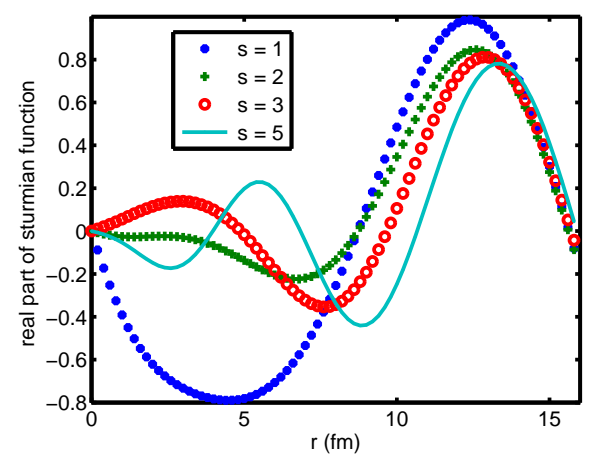

FIG. 6: Real parts of sturmian functions $\Phi_{s}$ for the potential $V_{P}$, for $k=0.5 \mathrm{fm}^{-1}$. This potential, defined in Table 【, has a repulsive core.

regions of the potential well. Examples are given in Figs. 6] and 7, In category II the real parts of $\Lambda$ are negative so as to turn the repulsive piece of the potential near the origin into an attractive well, and the formerly attractive valley into a repulsive barrier. Examples of the corresponding sturmian for indices $s=4,7,10,15$, and two of these functions are shown in Figs. 8 and 9, For $0<r<4 \mathrm{fm}$ the function $\Phi_{7}$ has a magnitude less than $10^{-4}$. The sturmian for $s=10$ is similar to that for $s=4$ in that it is also large near the origin (with an amplitude of $\simeq 10^{9}$ ) and has a node near $r=1$. The functions for $s=4$ and 10 are "resonant" in the radial region $r \in[0,4]$, while the one for $s=7$ is non-resonant. For a larger energy the effect of the barrier for the functions of class II decreases, and the absolute value of the eigenvalues $\eta_{s}=1 / \Lambda_{s}$ decreases for $s<10$, as is illustrated if Fig. 10. As will be shown in section III the functions for which $\eta_{s}<1$ play an important role in the Q-P method for the iterative correction of the truncation errors. Further, in the expansion of a 


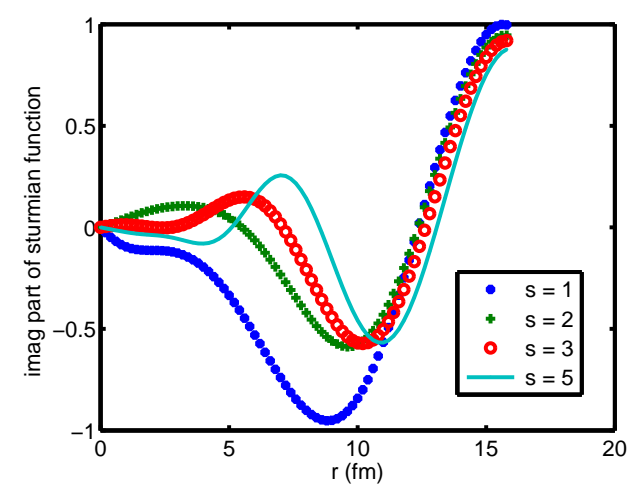

FIG. 7: Same as Fig. 6 for the imaginary parts of the sturmian functions

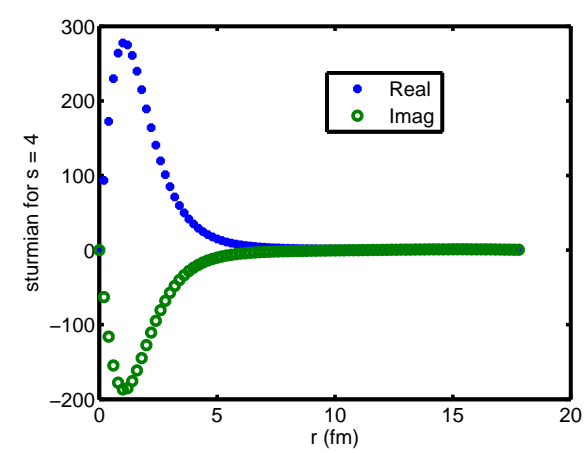

FIG. 8: Sturmian function in category II for $s=4$, for potential $V_{P}$ and $k=0.5$.

wave function in terms of sturmians, such as described in section IV-A below, a dominator $\left(1-\eta_{s}\right)$ is likely to appear. For the values of $s$ for which $\operatorname{real}\left(\eta_{s}\right) \simeq 1$, and $\operatorname{imag}\left(\eta_{s}\right) \simeq 0$, the corresponding sturmians make a resonant contribution to the expansion. In Fig. 11 some of the $\eta_{s}$ are illustrated in the form of an Argand diagram for three values of $k$, which shows that for $s=7$ the values of $\eta_{7}$ for all three values of $k$ satisfy this resonance criterion by lying close to unity.

\section{APPROXIMATE QUASI PARTICLE METHODS $\mathcal{S}_{1}$ AND $\mathcal{S}_{2}$}

The original Q-P iteration procedure was developed for the solution of the $L-S$ Eq. (3), but is couched here in terms of a more general integral kernel $\mathcal{O}$ for a one dimensional 


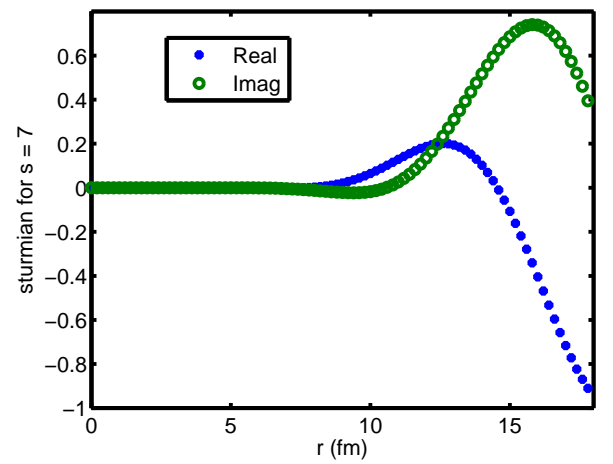

FIG. 9: Same as for Fig. 8, for $s=7$

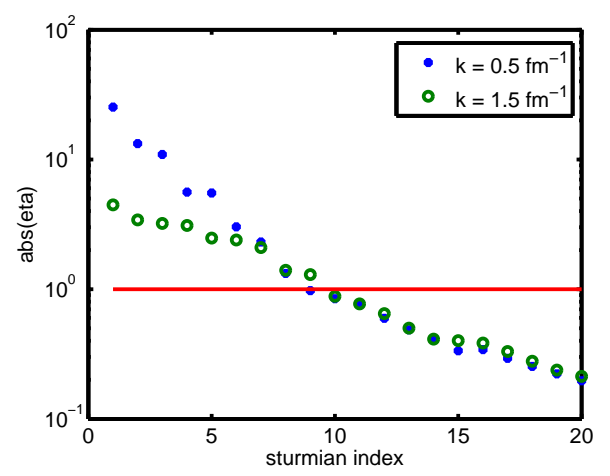

FIG. 10: Absolute value of $\eta_{s}$ as a function of the sturmian index $s$ for two values of the wave number $k$. The eigenvalues $\eta_{s}$ are defined in Eq. (10) with $\bar{V}=V_{P}$.

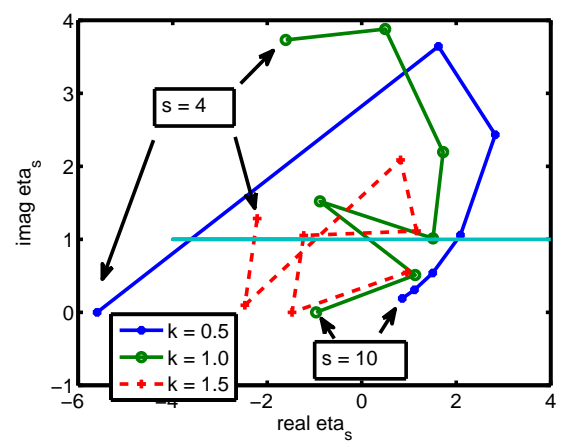

FIG. 11: Argand diagram of $\eta_{s}$, for the potential $V_{P}$ for $s=4,5, . .10$, for three values of $k$ (in units of $\left.\mathrm{fm}^{-1}\right)$. For $s=7$ they lie close to unity. 
integral equation

$$
\psi(r)=F(r)+\int_{0}^{\infty} \mathcal{O}\left(r, r^{\prime}\right) \psi\left(r^{\prime}\right) d r^{\prime} .
$$

This equation is to be solved for $\psi$, and $F$ is a known "driving" term. The shorthand form of Eq. (17) is

$$
\psi=F+\mathcal{O} \psi
$$

One procedure, convenient if $\mathcal{O}$ has a semi-separable nature, as is the case for Green's functions in configuration space, is to expand the unknown function into a set of Chebyshev polynomials, as is done in the IEM method [18], and obtain an answer to high accuracy without iterations. If however the operator is very complex and of large norm, so that a Born series would not converge, then an alternative method based on expansions into sturmian functions, described by Weinberg [13] as the "Quasi-Particle" method, is as follows. One approximates the operator $\mathcal{O}$ by a separable part of rank $N, \mathcal{O}_{N}$, and the remainder $\mathcal{O}_{R}$ is defined as

$$
\mathcal{O}_{R}=\mathcal{O}-\mathcal{O}_{N}
$$

and iterates on the remainder. If the norm of $\mathcal{O}_{R}$ is less than unity, the iterations should converge. Since the numerical complexity of performing iterations is less than solving a linear equation with a matrix of large dimension, Weinberg's method can be computationally advantageous. Weinberg's method to obtain $\mathcal{O}_{N}$ is to calculate the sturmians $\Gamma_{i}$ associated with the operator $\mathcal{O}$ which satisfy the same boundary conditions as $\psi$

$$
\mathcal{O} \Gamma_{i}=\gamma_{i} \Gamma_{i}
$$

and approximate $\mathcal{O}$ by a separable expansion into those sturmians $\Gamma_{i}$ whose eigenvalues $\gamma_{i}$, $i=1,2, . ., N$ have a magnitude larger than unity. Iterations on the remainder will then converge, since they involve powers of $\gamma_{i}$ which for $i>N$ are less than unity. A numerical example (example $P$ ) will be given for the case that $\mathcal{O}=\mathcal{G} V$, where the potential $V=V_{P}$ of Eq. (15) has a repulsive core and an attractive well.

Since the computational effort to obtain the eigenvectors and eigenvalues of a general operator $\mathcal{O}$ can be larger than the effort to solve Eq. (17) directly, a simplified method $\left(\operatorname{method} \mathcal{S}_{1}\right)$ will be described below, that uses auxiliary sturmian functions. These methods avoid the rigorous calculation of the $\Gamma_{i}$ by expanding the operator $\mathcal{O}$ into a finite set of auxiliary sturmian functions $\Phi_{s}, s=1,2, \ldots, N$, which satisfy Eq. (10) where $\bar{V}$ is an 
auxiliary potential, and which obey the same boundary conditions as the function $\psi$.

$$
\left.\mathcal{O}_{N}=\sum_{s=1}^{N} \mathcal{O} \Phi_{s}\right\rangle \frac{1}{\left\langle\Phi_{s} \bar{V} \Phi_{s}\right\rangle}\left\langle\Phi_{s} \bar{V}\right.
$$

These sturmian functions can be obtained reliably and with high accuracy, using a spectral expansion method [19], [12]. However, the auxiliary sturmians are not used to obtain the eigenfunctions for the operator $\mathcal{O}$, as is done in other studies [4] where the emphasis was in obtaining the scattering resonances, but rather to set up a well converging iteration scheme. In method $\mathcal{S}_{1}$, designed to solve Eq. (17) iteratively one first defines a function $\mathcal{F}^{(N)}$ which is the solution of

$$
\mathcal{F}_{1}^{(N)}=F+\mathcal{O}_{N} \mathcal{F}_{1}^{(N)}
$$

Since $\mathcal{O}_{N}$ is given by the separable representation (21), a solution of Eq. (22) is of the form

$$
\left.\mathcal{F}_{1}^{(N)}=F+\sum_{s^{\prime}=1}^{N} \mathcal{O} \Phi_{s}\right\rangle c_{s^{\prime}}
$$

with the coefficients $c_{s}$ defined as

$$
c_{s}=\frac{1}{\left\langle\Phi_{s} \bar{V} \Phi_{s}\right\rangle}\left\langle\Phi_{s} \bar{V} \mathcal{F}_{1}^{(N)}\right\rangle, \quad s=1,2, . ., N
$$

These coefficients are obtained algebraically by solving the matrix equation (25) for the $c_{s}$

$$
\sum_{s^{\prime}=1}^{N}\left(\delta_{s, s^{\prime}}-\mathcal{B}_{s . s^{\prime}}\right) c_{s^{\prime}}=\frac{1}{\left\langle\Phi_{s} \bar{V} \Phi_{s}\right\rangle}\left\langle\Phi_{s} \bar{V} F\right\rangle, \quad s=1,2, . ., N
$$

with

$$
\mathcal{B}_{s, s^{\prime}}=\frac{1}{\left\langle\Phi_{s} \bar{V} \Phi_{s}\right\rangle}\left\langle\Phi_{s} \bar{V} \mathcal{O} \Phi_{s^{\prime}}\right\rangle
$$

This equation is obtained [4] by multiplying Eq. (23) on the left by $\Phi_{s}(r) \bar{V}(r) /\left\langle\Phi_{s} \bar{V} \Phi_{s}\right\rangle$ and integrating over $r$ from 0 to $\infty$. The iterative solution of Eq. (17)

$$
\psi=\mathcal{F}_{1}^{(N)}+\chi^{(1)}+\chi^{(2)}+\chi^{(3)}+\ldots
$$

is obtained by solving

$$
\chi^{(n+1)}=\mathcal{O}_{N} \chi^{(n+1)}+\mathcal{O}_{R} \chi^{(n)}, \quad n=1,2, \ldots
$$

iteratively with $\chi^{(1)}=\mathcal{F}_{1}^{(N)}$. This series will converge if the norm of $\mathcal{O}_{R}$ is less than unity. The advantage of this procedure is that the functions $\chi^{(n)}$ can also be obtained algebraically, 
using the same matrix as required for the calculation of the coefficients $c_{s}$, and the evaluation of $\mathcal{O}_{R} \chi^{(n)}$ only involves integrals over known functions, rather than the solution of linear equations, which would be more computer intensive. The matrices are of dimension $N \times N$, where $N$ is of order 50 or less. Numerical examples are given below.

In the approximate Q-P method $\mathcal{S}_{2}$ one solves the once iterated form of Eq. (18) $\psi=$ $F+\mathcal{O}(F+\mathcal{O} \psi)$

$$
\psi=F+\mathcal{O} F+\mathcal{O}^{2} \psi
$$

The iterative treatment is similar to that of method $\mathcal{S}_{1}$, with the driving function $F$ replaced by $F+\mathcal{O} F$, and the operator $\mathcal{O}$ in Eq. (18) replaced by $\mathcal{O}^{2}$. This method lends itself to a decomposition of the matrices of $\mathcal{O}^{2}$ in terms of the singular value decomposition method (SVD) [22], [23], that takes the place of finding the eigenvalues and eigenvectors $\gamma_{i}$ and $\Gamma_{i}$ of the operator $\mathcal{O}$, and provides a measure of the norm of $\left(\mathcal{O}^{2}\right)_{R}$. This method is described in Ref. [24], but is not used for the numerical calculations described in the text.

\section{NUMERICAL EXAMPLES FOR METHODS $\mathcal{S}_{1}$ AND $\mathcal{S}_{2}$}

The operator $\mathcal{O}\left(r, r^{\prime}\right)$ for all of the three numerical examples described below is $\mathcal{G}_{0}\left(r, r^{\prime}\right) V\left(r^{\prime}\right)$, where $\mathcal{G}_{0}$ is the Green's function defined in Eqs. (44) and (55), $V$ is the scattering potential given by $V_{P}$, defined in Eq. (15) and Table I. The integral equation being solved is the conventional Lippmann-Schwinger $(L-S)$ scattering equation, Eq. (3). For most cases the wave number $k$ that defines the energy $E=k^{2}$ in Eq. (2) has the value $k=0.5 \mathrm{fm}^{-1}$. As illustrated in Fig. 1, $V_{P}$ has a repulsive core near the origin followed by an attractive well that decreases exponentially with distance. The original $Q-P$ method is illustrated by example $P$, and the approximate Q-P method $\mathcal{S}_{2}$ is illustrated for two sturmian potentials $V_{S}$ and $V_{W S}$. The size $N$ of the sturmian basis is 46,50 , and 41 for potentials $V_{S}$, $V_{B}$, and $V_{W S}$, respectively

\section{A. Q-P example "P"}

In this case the sturmian potential is equal to the scattering potential, $\bar{V}=V_{P}$, and hence the sturmian functions $\Phi_{s}$, defined by Eq. (10) are the same, to within a normalization

constant, as the eigenfunctions $\Gamma_{i}^{(N)}$ of the operator $\mathcal{O}=\mathcal{G}_{0} V_{P}$, and the eigenvalues $\eta_{s}$ are also 
the same as the eigenvalues $\gamma_{i}^{(N)}$ The $\Phi_{s}$ used in the present examples are normalized such that asymptotically they equal the function $H(r)$, with unit coefficient. If an eigenfunction $\bar{\Phi}_{s}$ is normalized differently, then it can be renormalized according to

$$
\Phi_{s}=\frac{-\eta_{s} k}{\left\langle F V \bar{\Phi}_{s}\right\rangle} \bar{\Phi}_{s}
$$

where $\left\langle F V \bar{\Phi}_{s}\right\rangle=\int_{0}^{r_{\max }} F(r) V(r) \bar{\Phi}_{s}(r) d r$. The value of $k$ is $0.5 \mathrm{fm}^{-1}$, and $r_{\max }=30 \mathrm{fm}$. Some of these sturmians are illustrated in Figs. 6] to 9 and the absolute values of $\eta_{s}$ are displayed in Fig. 10. The operator $\mathcal{O}_{N}$ becomes

$$
\mathcal{O}_{N}=\sum_{s=1}^{N} \Phi_{s}(r) \frac{\eta_{s}}{\left\langle\Phi_{s} V \Phi_{s}\right\rangle} \Phi_{s}\left(r^{\prime}\right) V\left(r^{\prime}\right) .
$$

In the present example $N=26$, and $\left|\eta_{26}\right|=0.1425$. The matrix elements in Eq. (25) become diagonal, the coefficients $c_{s}$, defined in Eq. (25), are given by $\left\langle F V \Phi_{s}\right\rangle /\left[\left(1-\eta_{s}\right)\left\langle\Phi_{s} V \Phi_{s}\right\rangle\right]$, and the function $\mathcal{F}^{(N)}$, solution of Eq. (22), is given by

$$
\mathcal{F}_{1}^{(N)}(r)=F(r)+\sum_{s=1}^{N} \frac{\eta_{s}}{1-\eta_{s}} \frac{\left\langle F V \Phi_{s}\right\rangle}{\left\langle\Phi_{s} V \Phi_{s}\right\rangle} \Phi_{s}(r),
$$

The quantity $S_{1}$ is defined by the asymptotic limit of $\mathcal{F}$

$$
\mathcal{F}_{1}^{(N)}(r \rightarrow \infty)=F(r)+S_{1} H(r) .
$$

and is given by $S_{1}=\sum_{1}^{M} \bar{c}_{s}$, where

$$
\bar{c}_{s}=\frac{\eta_{s}}{1-\eta_{s}} \frac{\left\langle F V \Phi_{s}\right\rangle}{\left\langle\Phi_{s} V \Phi_{s}\right\rangle}
$$

and the iterative corrections to $\psi$ are

$$
\psi=\mathcal{F}_{1}^{(N)}+\mathcal{O}_{R} F+\mathcal{O}_{R}^{2} F+\mathcal{O}_{R}^{3} F+\ldots
$$

as shown in the Appendix A.

The values of $\bar{c}_{i}$ are shown in Fig. 12. They begin to decrease nearly monotonically for $i>15$, but the rate of decrease is slow. For example $\bar{c}_{15}=-0.7017+1.0067 i$, and $\bar{c}_{26}=-0.0107+0.0395 i$. This shows that without the additional iterations described next, the accuracy of $S$ given by $S_{1}$ alone, would be good to only one or two significant figures. For example, the value of $S_{1}$ obtained from $\mathcal{F}^{(N)}$ is $S_{1}=0.7075982522+0.493097515 i$, while the "exact" value of $S$, obtained via the spectral Integral Equation Method (IEM) [18] is $S=0.34016623828+0.8664518117 i$. In Fig. 13 the improvement due to the successive Weinberg iterations are displayed. 


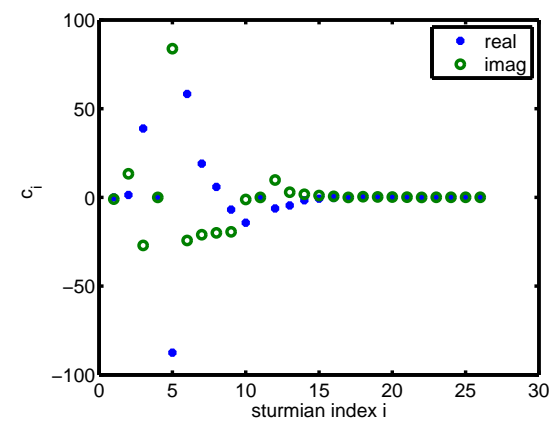

FIG. 12: The coefficients $\bar{c}_{i}$ as defined in Eq. (34) for the Q-P method. The sturmian potential is the same as the scattering potential $V_{P}$ and $k=0.5 \mathrm{fm}^{-1}$.

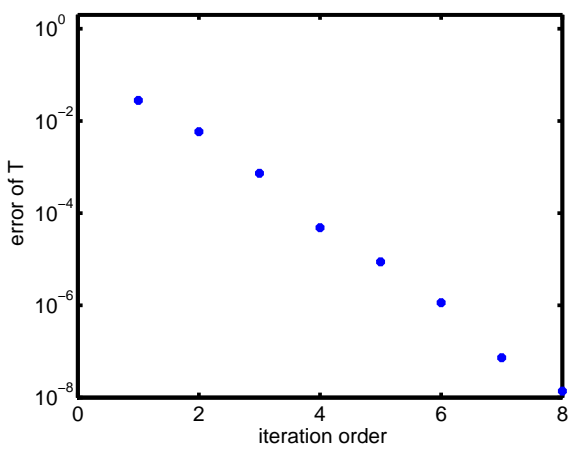

FIG. 13: The iterative improvement of the asymptotic value of $S$ of the scattering wave function $\psi$ is shown as the absolute value of the difference between the iterative value of $S$, obtained from Eq. (35) and the value of $\psi$, obtained by solving the Lippmann-Schwinger scattering equation directly. The first point illustrates the difference between the asymptotic values of $\mathcal{F}$ and $\psi$, while the subsequent points illustrate the contributions of the successive Weinberg iterations.

\section{B. Examples for the approximate Q-P Methods $\mathcal{S}_{1}$ and $\mathcal{S}_{2}$.}

For both methods $\mathcal{S}_{1}$ and $\mathcal{S}_{2}$ the operator $\mathcal{O}$ is again $\mathcal{G}_{0} V$, with $V=V_{P}$, and the sturmian potential $\bar{V}$ can be either $V_{S}$, Eqs. (15), or $V_{B}$, the latter defined in Appendix $B$. In this section the normalization of the sturmian functions is such that

$$
\left\langle\Phi_{s} \bar{V} \Phi_{s}\right\rangle=\eta_{s}, s=1,2, . ., N
$$

For method $\mathcal{S}_{1}$ the elements of the matrix $\mathcal{B}$, Eq. (26) and all other equations described in section III apply. The separable approximation to the 
operator $\mathcal{O}$, Eq. (21), is given by

$$
\left.\mathcal{O}_{N}=\sum_{s=1}^{N} \mathcal{G}_{0} V \Phi_{s}\right\rangle \frac{1}{\left\langle\Phi_{s} \bar{V} \Phi_{s}\right\rangle}\left\langle\Phi_{s} \bar{V}\right.
$$

and as a result of the normalization (36) the $n$ 'th power of $\mathcal{O}_{N}$ is

$$
\left.\left(\mathcal{O}_{N}\right)^{n}=\sum_{s, s^{\prime}=1}^{N} \mathcal{G}_{0} V \Phi_{s}\right\rangle\left(\boldsymbol{V}^{n-1}\right)_{s, s^{\prime}} \frac{1}{\eta_{s^{\prime}}}\left\langle\Phi_{s^{\prime}} \bar{V}\right.
$$

where the matrix $\boldsymbol{V}$ has the matrix elements

$$
\boldsymbol{V}_{s, s^{\prime}}=\left\langle\Phi_{s} V \Phi_{s^{\prime}}\right\rangle
$$

Equation (38) shows that if the norm of the matrix $\boldsymbol{V}$ is larger than unity, iterations performed with $\left(\mathcal{O}_{N}\right)^{2}$ will not converge. For this reason separating from $\boldsymbol{V}$ a part that has a norm less than unity, and performing iterations for $\left(\mathcal{O}_{N}\right)^{2}$ on that part, will converge. That is the reason for solving the iterated equation (29) rather than the original Eq. (3), as will be explained further below. For more complicated integral kernels a similar separation is also feasible with the Singular Value Decomposition method (SVD) [24] but is not needed for the examples given here.

In method $\mathcal{S}_{2}$ one decomposes $\mathcal{O}^{2}$, the square of the full operator, into two parts $\mathcal{O}^{2}=$ $\mathcal{O}_{N}^{2}+\mathcal{O}_{R}^{2}$. According to Eq. (38) $\left(\mathcal{O}_{N}\right)^{2}$ is given by

$$
\left.\mathcal{O}_{N}^{2}=\sum_{s, s^{\prime}=1}^{N} \mathcal{G}_{0} V \Phi_{s}\right\rangle \boldsymbol{V}_{s, s^{\prime}} \frac{1}{\eta_{s^{\prime}}}\left\langle\Phi_{s^{\prime}} \bar{V}\right.
$$

and one $\mathcal{O}_{R}^{2}$ is defined as

$$
\mathcal{O}_{R}^{2}=\mathcal{O}^{2}-\mathcal{O}_{N}^{2}
$$

In order to solve the once iterated $L-S$ equation (29), one defines the function $\mathcal{F}_{2}$ as the solution of

$$
\mathcal{F}_{2}^{(N)}=F+\mathcal{O} F+\mathcal{O}_{N}^{2} \mathcal{F}_{2}^{(N)}
$$

given in the present case by

$$
\left.\mathcal{F}_{2}^{(N)}=F+\mathcal{O} F+\sum_{s, s^{\prime}=1}^{N} \mathcal{G}_{0} V \Phi_{s}\right\rangle \boldsymbol{V}_{s, s^{\prime}} c_{s^{\prime}}^{(2)}
$$


where $c_{s^{\prime}}^{(2)}=\left\langle\Phi_{s^{\prime}} \bar{V} \mathcal{F}_{2}^{(N)}\right\rangle / \eta_{s^{\prime}}$ is the solution of the linear equation

$$
\sum_{s^{\prime}=1}^{N}\left(\delta_{s, s^{\prime}}-\boldsymbol{V}_{s, s^{\prime}}^{2}\right) c_{s^{\prime}}^{(2)}=\frac{1}{\eta_{s}}\left\langle\Phi_{s} \bar{V}(F+\mathcal{O} F)\right\rangle .
$$

The terms $\chi_{2}^{(n)}$ required for the subsequent iterations,

$$
\psi=\mathcal{F}_{2}^{(N)}+\chi_{2}^{(2)}+\chi_{2}^{(3)}+\chi_{2}^{(4)}+\ldots
$$

are obtained by solving

$$
\chi_{2}^{(n+1)}=\mathcal{O}_{N}^{2} \chi_{2}^{(n+1)}+\mathcal{O}_{R}^{2} \chi_{2}^{(n)}, \quad n=1,2, \ldots
$$

with $\chi^{(1)}=\mathcal{F}_{2}^{(N)}$.

A justification for the advantage of method $\mathcal{S}_{2}$ over $\mathcal{S}_{1}$ is as follows. Formally, Eq. (46) can be written as $\chi_{2}^{(n+1)}=\left[\left(1-\mathcal{O}_{N}^{2}\right)^{-1} \mathcal{O}_{R}^{2}\right]^{n} \mathcal{F}_{2}^{(N)}$, and hence the rate of convergence of the iterations depends on the norm of the operator $\left(1-\mathcal{O}_{N}^{2}\right)^{-1} \mathcal{O}_{R}^{2}$. Since the norm of $\left(1-\mathcal{O}_{N}^{2}\right)^{-1}$ is expected to be smaller than unity, the rate of convergence should be faster than the powers of the norm of $\mathcal{O}_{R}^{2}$. In view of the completeness of the sturmian functions, $\mathcal{O}_{R}^{2}$ is also given by

$$
\left.\mathcal{O}_{R}^{2}=\sum_{s, s^{\prime}=N+1}^{\infty} \mathcal{G}_{0} V \Phi_{s}\right\rangle \boldsymbol{V}_{s, s^{\prime}} \frac{1}{\eta_{s^{\prime}}}\left\langle\Phi_{s^{\prime}} \bar{V}\right.
$$

By defining $\tilde{\boldsymbol{V}}$

$$
\begin{gathered}
\tilde{\boldsymbol{V}}_{s, s^{\prime}}=\boldsymbol{V}_{s, s^{\prime}} \quad s, s^{\prime}>N \\
\tilde{\boldsymbol{V}} s, s^{\prime}=0 \quad s, s^{\prime} \leq N
\end{gathered}
$$

and keeping in mind that

$$
\frac{1}{\eta_{s^{\prime}}}\left\langle\Phi_{s^{\prime}} \bar{V} \mathcal{G}_{0} V \Phi_{s}\right\rangle=\boldsymbol{V}_{s^{\prime}, s}
$$

one can show that the powers of $\mathcal{O}_{R}^{2}$ are given by

$$
\left.\left(\mathcal{O}_{R}^{2}\right)^{n}=\sum_{s, s^{\prime}=N+1}^{\infty} \mathcal{G}_{0} V \Phi_{s}\right\rangle\left(\tilde{\boldsymbol{V}}^{2 n-1}\right)_{s, s^{\prime}} \frac{1}{\eta_{s^{\prime}}}\left\langle\Phi_{s^{\prime}} \bar{V}\right.
$$

and hence the norm of the iterations should decrease with the order of the iteration $n$ as

$$
\left|\chi_{2}^{(n)}\right|<\mathfrak{k}\left|\tilde{\boldsymbol{V}}^{2 n-3}\right|, \quad n=1,2, . .
$$

where $\mathfrak{k}$ is some constant. 


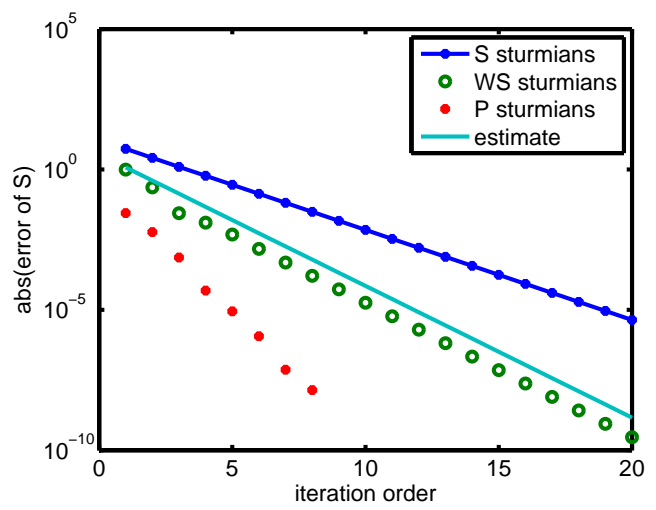

FIG. 14: Iterative rate of convergence of the asymptotic limit of the wave function to the "exact" one. The sturmian functions $S$ and $W S$ are obtained with potentials $V_{S}$ and $V_{W S}$, respectively, which have no repulsive core. Iteration method $\mathcal{S}_{2}$ was used for results $V_{S}$ and $V_{W S}$ and method $\mathcal{S}_{1}$ was used for potential $V_{P}$. (The latter is identical to the potential used to calculate the scattering wave function). The solid line represents $(0.66)^{p}$, with $p=2.6 \times n-3, n$ being the iteration order. The wave number is $k=0.5 \mathrm{fm}^{-1}$.

By contrast, for method $\mathcal{S}_{1}$

$$
\left.\left(\mathcal{O}_{R}\right)^{n}=\sum_{s, s^{\prime}=N+1}^{\infty} \mathcal{G}_{0} V \Phi_{s}\right\rangle\left(\tilde{\boldsymbol{V}}^{n-1}\right)_{s, s^{\prime}} \frac{1}{\eta_{s^{\prime}}}\left\langle\Phi_{s^{\prime}} \bar{V}\right.
$$

and by a reasoning similar to the one above, one expects

$$
\left|\chi^{(n)}\right|<\mathfrak{k}^{\prime}\left|\tilde{\boldsymbol{V}}^{n-1}\right|, \quad n=1,2, . .
$$

These convergence estimates are supported by Figs. 15] and 20, Although one would expect from Eq. (51) that the magnitude of the iteration error decreases as the square of the iteration order $n$, (i.e. a power 2) the fit in Fig. 15 decreases by a larger power, 2.6. Nevertheless, the convergence of method $\mathcal{S}_{1}$ is considerably slower than for $\mathcal{S}_{2}$, as illustrated in Fig. 20, and is in accordance with Eq. (53).

The results for method $\mathcal{S}_{2}$, displayed in Fig. (14), also show that the iterations using sturmians that are based on a potential with a longer range, $V_{W S}$, than the potential $V_{S}$ converge faster. This feature can be understood from the fact that at large distances the sturmians become linearly dependent, since asymptotically they become proportional to each other, as can be seen from Figs. 4 to 7, and hence at large distances they do not form 


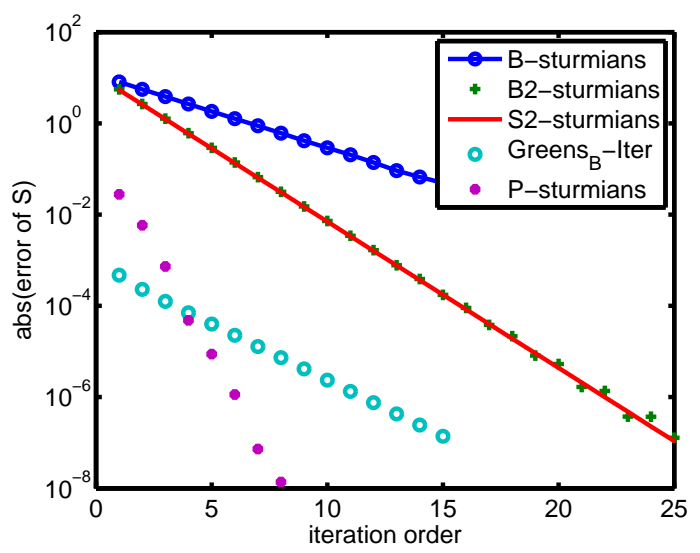

FIG. 15: The convergence of the iterations $n=1,2, .$. as measured by the error of the asymptotic value of the wave function $\psi$, Eq. (6) . The results labeled $B$ and $B 2$ are obtained with sturmian potential $\bar{V}=V_{B}$ for methods $\mathcal{S}$ and $\mathcal{S}_{2}$, respectively. The solid line labeled as $\mathrm{S} 2$ is obtained with method $\mathcal{S}_{2}$ for the sturmian potential $V_{S}$. The result $P$ is obtained with the original Q-P method, with sturmians obtained for potential $V_{P}$. The open circles, labeled Green $_{B}$, are obtained with Green's function iterations, based on $V_{P}-V_{B}$, described in Appendix $B$.

a good basis set. Indeed, at short distances the convergence to the scattering wave function is much faster than at large distances, as can be seen from Fig. 16, obtained with sturmians for $V_{S}$.

The results labeled $B$ and $B 2$ in Fig. 15 are obtained with methods $S$ and $S_{2}$, respectively, for sturmians based on potential $V_{B}$. This potential, defined in Eq. (16) and further illustrated in the Appendix, is identical to potential $V_{P}$ at large distances, but has its repulsive core decreased by a factor that decreases to zero near the origin. Both potentials $V_{B}$ and $V_{S}$ decay in a similar fashion at large distances while $V_{B}$ has a barrier near the origin, and $V_{S}$ does not. The result that both sets of sturmians give nearly indistinguishable results for the iteration, as shown in Fig. 15, shows that the behavior of the sturmians near the origin does not significantly affect the results. However, using the sturmians defined with the longer range potential $V_{W S}$ the convergence is significantly better at large distances, as is shown in Fig. 17,

The open circles in Fig. 20, labeled as Greens $s_{B}$ were obtained with a Green's function iteration method, described in Appendix $B$, in which potential $V_{p}$ is divided into $V_{B}+$ $\left(V_{P}-V_{B}\right)$. The $L-S$ equation with potential $V_{B}$ is solved to produce the function $\mathcal{F}$ 


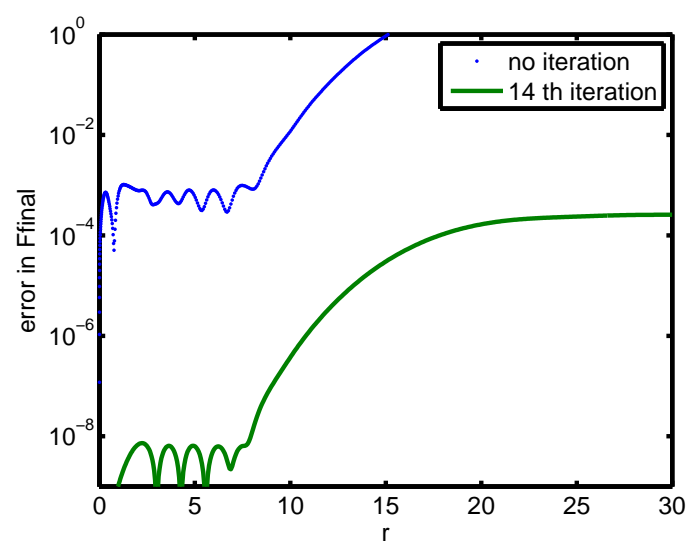

FIG. 16: Absolute value of the error of the wave function $\psi$ as a function of radial distance $r$. The result labeled "no iteration" illustrates the error of the function $\mathcal{F}_{2}^{(N)}$, Eq. (43), with $N=46$. The other line is obtained after the 14 'th iteration. These resuts are obtained with method $\mathcal{S}_{2}$ using the sturmians for potential $V_{S}$. The scattering wave function $\psi$ is obtained with potential $V_{P}$ for $k=0.5 \mathrm{fm}^{-1}$.

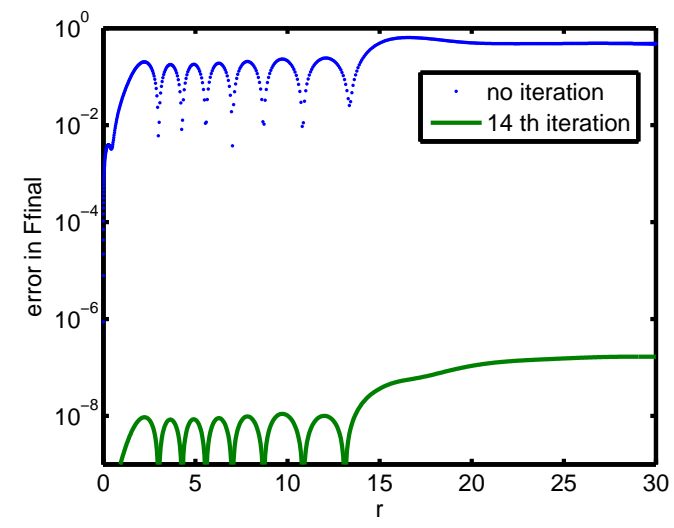

FIG. 17: Same as Fig. 16, using 41 sturmians based on the $V_{W S}$ potential.

and the corrections due to $\left(V_{P}-V_{B}\right)$ are obtained iteratively in a Born-series manner as approximation to the exact function $\psi$. The asymptotic value of $\mathcal{F}$ is much closer to that of $\psi$ than for method $\mathcal{S}_{2}$, but the rate of convergence of the Green's function iterations is not as fast as that of method $\mathcal{S}_{2}$. 


\section{SUMMARY AND CONCLUSIONS}

The main result of this paper is to present a viable iteration procedure to correct for the truncation errors of a sturmian expansion used for the solution of an integral equation. The basic idea is to construct a separable (and truncated) approximation to the integral kernel of the integral equation, that leads to an algebraic solution for the approximated integral equation, denoted as $\mathcal{F}$. The corrections to $\mathcal{F}$ are performed iteratively, and the rate of convergence of the iteration is investigated. The advantage of doing iterations rather than solving the integral equation numerically directly, is that the numerical complexity for obtaining the algebraic solution for $\mathcal{F}$ and the subsequent iterations can be substantially less than for the direct numerical solution, especially if the kernel of the integral equation is very complicated. An example would be the solution of a Schrödinger equation with nonlocal potentials, recast into a Lippmann-Schwinger $(L-S)$ integral form. The numerical examples are done in configuration space for a one-dimensional $(L-S)$ equation for which the integral kernel is of the form $\mathcal{G}_{0} V$, where $\mathcal{G}_{0}$ is the undistorted Green's function and $V$ the scattering potential, and the scattering energy is positive. However the formalism can be generalized to more general integral kernels. Several methods are compared. One is the "classical" quasi-particle [13] method, that requires a basis of sturmians that are eigenfunctions of the

kernel of the integral equation. For the application of this method to the scattering of a particle from a potential with a repulsive core, $V_{P}$, the iterations converge very fast (denoted as method $P$ ) and to high accuracy, as illustrated in Figs. 13, 15, and 20, If the sturmian eigenstates of the above mentioned integral kernel are not available, as is assumed here, then a set of auxiliary sturmian functions are introduced, and the convergence of the iterations is examined. Two methods are presented for this case. In method $\mathcal{S}_{1}$ the iterative solution of the original integral equation is examined, while in method $\mathcal{S}_{2}$ the solution of a once iterated integral equation is considered. It is predicted and also found that the iterations of method $\mathcal{S}_{2}$ converge faster than for method $\mathcal{S}_{1}$, and both methods converge faster than the iterations performed with a Green's function Born approximation procedure, but more slowly than the original Q-P method. A key condition for obtaining an accurate approximation to the wave function at large distances is that the auxiliary sturmian functions be based on an auxiliary potential whose range is sufficiently larger than that of the scattering potential. An alternate method for solving the once iterated (but complicated) integral equation, based 
on the singular value decomposition of a matrix, is described in Appendix $C$ but not used in the numerical examples.

An accuracy of at least eight significant figures is obtained for all the numerical calculations performed. This accuracy is achieved by using the spectral method for solving integral equations [19], both for the scattering wave function and for the sturmian functions. As a result, previous investigations with positive energy sturmians [21], hampered by lack of this type of accuracy, were improved upon and expanded in the present study.

\section{Appendix A}

A proof of Eq. (35) is as follows: The operator $\mathcal{O}_{N}$, given by Eq. (31), projects any function into the space spanned by the sturmians $\Phi_{s}, s=1,2, . ., N$, while the operator $\mathcal{O}_{R}$ projects any function into the sturmian space for $s=N+1, N+2, \ldots$ The latter property follows from the orthogonality given by Eq. (12), and the fact that the sturmians form a complete set. Hence $\mathcal{O}_{R}$, Eq.(19), can also be written as

$$
\mathcal{O}_{R}=\sum_{N+1}^{\infty} \Phi_{s}\left[\eta_{s} /\left\langle\Phi_{s} V \Phi_{s}\right\rangle\right]\left\langle\Phi_{s} V\right.
$$

This expression for $\mathcal{O}_{R}$ also shows that the norm of $\mathcal{O}_{R}$ is less than unity if $\left|\eta_{s}\right|<1$ for $s>N$. In view of Eqs. (22), and (154) one finds that $\mathcal{O}_{R} \mathcal{F}_{1}^{(N)}=\mathcal{O}_{R} F$. By writing

$$
\psi=\mathcal{F}_{1}^{(N)}+\chi
$$

and by by making use of Eqs. (28) for $n=1,\left(1-\mathcal{O}_{N}\right) \chi^{(1)}=\mathcal{O}_{R} \mathcal{F}^{(N)}=\mathcal{O}_{R} F$. Hence $\chi^{(1)}$ lies entirely in the sturmian space with $s>N$ and $\chi^{(1)}=\mathcal{O}_{R} F$. In view of Eq. (28) the same argument can be made for $\chi^{(2)}, \chi^{(3)}$.. and Eq. (35) is proven for the case that $\mathcal{O}=G_{0} V$ and $\bar{V}=V$.

\section{Appendix B: The Green's function iterations.}

At large distances potential $V_{B}$ is identical to $V_{P}$, but at short distances the repulsive core is reduced by the factor $f(r)=1-\exp \left[(r / 0.5)^{2}\right]$, as given by Eq. (16)

$$
V_{B}(r)=f(r) \times V_{P}(r)
$$

This potential is illustrated by the open circles in Fig. 18 This potential was defined for the purpose of performing a Green's function iteration, as follows. The potential $V_{P}$ is divided 


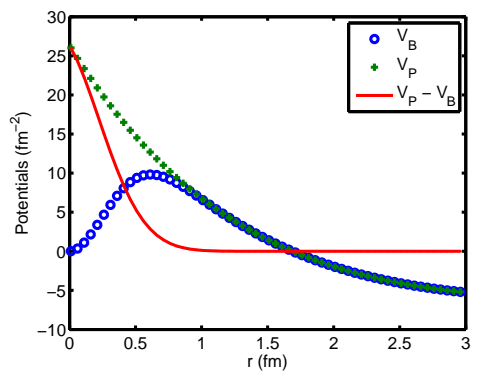

FIG. 18: The potental $V_{B}$ is illustrated by the open circles, and is defined in Eq. (56). The purpose of this potential is to eliminate the repulsive core of potential $V_{P}$, the latter illustrated by the + symbols. The difference between these two potentials, illustrated by the solid line, serves to perform iterations with a Green's function Born-type method.

into two parts $V_{B}$ and $V_{2}=\left(V_{P}-V_{B}\right)$. The first part has a suppressed repulsive core, and the second part, $V_{2}$ adds the repulsive core again. The iterations are based on Green's functions that explicitly include the distortion due to $V_{B}$, and iterate over the effect of $V_{2}$ in a fashion similar to the Born approximation. The Green's function method has many applications, but in view of the factor $1 / k$ in the Greens functions $F$ and $H$, Eq. (4), this method does not converge well [22] for low values of the wave number $k$.

\section{Appendix C: Iterative method to calculate Sturmian eigenfunctions.}

In what follows the eigenvalue subscripts $s$ will be dropped, and the iterations which lead to the solution for a particular fixed value of $s$ will be described. In the differential equation (8) the exact values of $\Lambda$ and $\Phi$ are initially unknown, but the equation can still be solved for a guessed value $\Lambda^{(1)}$. One solutions, denoted as $Y(r)$ satisfies the correct boundary conditions at the origin, and is integrated from inside outward.

$$
\mathcal{D} Y=\Lambda^{(1)} \bar{V} Y, \quad 0 \leq r \leq R_{I}
$$

The other solution, denoted as $Z(r)$, satisfies the correct boundary conditions asymptotically,

$$
\mathcal{D} Z=\Lambda^{(1)} \bar{V} Z, \quad R_{I} \leq r \leq \infty
$$

and is integrated inward towards the intermediate point $R_{I}$ chosen to lie within the region where $\bar{V}$ is non negligible. The three solutions at the point $R_{I}$ are denoted as $\Phi_{I}, Y_{I}$, and 
$Z_{I}$. One can renormalize $Y$ by a factor $\mathfrak{k}=Z_{I} / Y_{I}$

$$
\bar{Y}(r)=\mathfrak{k} Y(r)
$$

so that $\bar{Y}_{I}=Z_{I}$.

If one multiplies Eq. (8) on both sides by $\bar{Y}$, and Eq. (57) by $\Phi$, integrates over $r$ from 0 to $R_{I}$, and subtracts the results one from the other, after an integration by parts one obtains

$$
\left.\left(\bar{Y} \Phi^{\prime}-\bar{Y}^{\prime} \Phi\right)\right|_{0} ^{R_{I}}=\left(\Lambda-\Lambda^{(1)}\right) \int_{0}^{R_{I}} \bar{Y}(r) \bar{V}(r) \Phi(r) d r,
$$

where the prime denotes a derivative with respect to $r$. By a similar procedure one obtains

$$
\left.\left(Z \Phi^{\prime}-Z^{\prime} \Phi\right)\right|_{R_{I}} ^{\infty}=\left(\Lambda-\Lambda^{(1)}\right) \int_{R_{I}}^{\infty} Z(r) \bar{V}(r) \Phi(r) d r .
$$

By adding Eqs. (60) and (61), by noting that $\left.\left(\bar{Y} \Phi^{\prime}-\bar{Y}^{\prime} \Phi\right)\right|_{0}=0$ because $\bar{Y}$ and $\Phi$ vanish at the origin, and that $\left.\left(Z \Phi^{\prime}-Z^{\prime} \Phi\right)\right|^{\infty}=0$ because $Z$ and $\Phi$ obey the same boundary condition asymptotically (to within a normalization constant), one obtains

$$
\Phi_{I}\left(Z_{I}^{\prime}-\bar{Y}_{I}^{\prime}\right)=\left(\Lambda-\Lambda^{(1)}\right)\left[\int_{0}^{R_{I}} \bar{Y}(r) \bar{V}(r) \Phi(r) d r+\int_{R_{I}}^{\infty} Z(r) \bar{V}(r) \Phi(r) d r\right] .
$$

The above equation is still rigorously valid. The approximation consists in replacing $\Phi(r)$ in the first integral by $\bar{Y}(r)$, and by $Z(r)$ in the second integral, and further, by replacing $\Phi_{I}$ in the left hand side by either $\bar{Y}_{I}$ or $Z_{I}$. After making these approximations, and by dividing both sides of Eq. (62) by $\left(\bar{Y}_{I}\right)^{2}=\left(Z_{I}\right)^{2}$, one obtains the final result

$$
\Lambda^{(2)}=\Lambda^{(1)}+\frac{Z_{I}{ }^{\prime} / Z_{I}-Y_{I}^{\prime} / Y_{I}}{\left(\int_{0}^{R_{I}} Y^{2}(r) \bar{V}(r) d r\right) / Y_{I}^{2}+\left(\int_{R_{I}}^{\infty} Z^{2}(r) \bar{V}(r) d r\right) / Z_{I}^{2}}
$$

In the above, $\Lambda^{(2)}$ replaces $\Lambda$ as the iteratively corrected value for $\Lambda^{(1)}$; also the factor $\mathfrak{k}$ disappeared because it canceled in the numerator and denominator of Eq. (63).

Equation (63) is the generalization to sturmian eigenvalues of the iterative calculation of energy eigenvalues, Eq. (7) of Ref. [20]. It can also be applied to negative energies, and can be generalized to the cases of coupling potentials or of non-local potentials, which however goes beyond the scope of the present study.

The functions $Y$ and $Z$ can be calculated as the solutions of the differential Eqs. (57) and (58) by any convenient finite difference method, or they can be obtained as the solutions of the integral equations

$$
Y(r)=F(r)+\Lambda^{(1)} \int_{0}^{R_{I}} \mathcal{G}_{0}\left(r, r^{\prime}\right) \bar{V}\left(r^{\prime}\right) Y\left(r^{\prime}\right) d r^{\prime}
$$


and

$$
Z(r)=H(r)+\Lambda^{(1)} \int_{R_{I}}^{\infty} \mathcal{G}_{0}\left(r, r^{\prime}\right) \bar{V}\left(r^{\prime}\right) Z\left(r^{\prime}\right) d r^{\prime}
$$

or else the whole iteration procedure can be bypassed by obtaining the eigenvalues $\eta_{s}$ and eigenfunctions $\Phi_{s}$ of the integral operator $\mathcal{G}_{0} \bar{V}$ contained in Eq. (10). In the numerical calculations described below, this latter procedure is carried out (with questionable accuracy

according to Ref [12]) so as to provide the initial values $\Lambda_{s}^{(1)}$ for the iteration, Eq. (63). The present implementation obtains the solution of Eqs. (64) and (65) by using the spectral Chebyshev expansion method, [18], [19] because its high accuracy can be predetermined by the specification of an accuracy parameter tol, and the method is by now well tested.

\section{Appendix D}

For future benchmark purposes, the eigenvalues of Eq. (8) are given in the Table [I]. The potential is $V_{S}$ defined in Eq. (15) and Table I, the wave number is $k=0.5 \mathrm{fm}^{-1}$.

[1] M. Rotenberg, Ann. of Phys. (N.Y.) 19, 262 (1962); M. Rotenberg, "Theory and Applications of Sturmian functions" in Adv. in Atomic and Molec. Phys., ed by D.R. Bates and I. Esterman, (Academic Press, N.Y. (1970)), vol 6, p233-268;

[2] S. Klarsfeld and A. Maquet, Phys. Lett. A 78, 40 (1980);

[3] E. Karule and A. Gailitis, J. Phys. B: At. Mol. Opt. Phys. 43,065601 (2010);

[4] K. Amos, L. Canton, G. Pisent, J. P. Svenne, D. van der Knijff, Nucl. Phys. A 728, 65 (2003); P. Fraser et al, Phys. Rev. Lett. 242501 (2008); U. Weiss, Nucl. Phys. A 156, 53 (1970);

[5] G. H. Rawitscher, D. Lukaszek, R.S. Mackintosh and S. G. Cooper, Phys. Rev. C 49, 1621 (1994);

[6] J. S. Ball and D. Wong, Phys. Rev. 169, 1362 (1968); G. H. Rawitscher and L. Canton, Phys. Rev. C 44, 60 (1991); L. Canton and G. H. Rawitscher, J. of Phys. G: Nucl. and Part. Phys. 17, 429 (1991);

[7] D. Eyre, H. G. Miller, Phys. Lett. B 153 B, 5, (1985); Z. C. Kuruoglu and F. S. Levin, Annals of Phys. 163,120 (1985); A. C. Fonseca and M. T. Pena, Phys. Rev. A 38, 4967 (1998); S. Yu and J. H. Macek, Phys. Rev. A 55, 3605 (1997); J. M. Randazzo, L. U. Ancarani, G. 


\begin{tabular}{|l||l|l|}
\hline & Real part of $\Lambda_{s}$ & Imag. part of $\Lambda_{s}$ \\
\hline 1 & -0.03297806784 & -0.05633093256 \\
\hline 2 & 0.04181033607 & -0.12298817955 \\
\hline 3 & -0.02140651197 & -0.23641901361 \\
\hline 4 & 0.21055103262 & -0.15935376881 \\
\hline 5 & 0.43743611684 & -0.17810671020 \\
\hline 6 & 0.71976101832 & -0.19289033454 \\
\hline 7 & 1.05649701588 & -0.20516494913 \\
\hline 8 & 1.44649394898 & -0.21538251834 \\
\hline 9 & 1.88887575723 & -0.22390738772 \\
\hline 10 & 2.38303461225 & -0.23106484062 \\
\hline 11 & 2.92855765503 & -0.23712639599 \\
\hline 12 & 3.52516147256 & -0.24230800967 \\
\hline 13 & 4.17264661513 & -0.24677772914 \\
\hline 14 & 4.87086853262 & -0.25066566005 \\
\hline 15 & 5.61971940360 & -0.25407296058 \\
\hline 16 & 6.41911669954 & -0.25707898962 \\
\hline 17 & 7.26899583793 & -0.25974669404 \\
\hline 18 & 8.16930533353 & -0.26212658464 \\
\hline 19 & 9.12000350403 & -0.26425965551 \\
\hline 20 & 10.1210561674 & -0.26617953514 \\
\hline 21 & 11.1724349888 & -0.26791408364 \\
\hline 22 & 12.2741162656 & -0.26948659119 \\
\hline
\end{tabular}

TABLE II: Eigenvalues for Sturmian potential $V_{S}$ for $k=0.5 \mathrm{fm}^{-1}$.

Gazaneo, A. L. Frapiccini and F. D. Colavecchia, Phys. Rev. A 81, 042520 (2010); A. Maquet, V. Veniard, T. A. Marian, J. Phys B 31, 3743 (1998);

[8] L. Hostler, J. Math. Phys. 5, 1235 (1964); ibid. 11, 2966 (1970); ibid. 26, 1348 (1985);

[9] A. Maquet, Phys. Rev. A 151088 (1977); E. Arnous, J. Bastian, and A. Maquet, Phys. Rev. A 27, 977 (1983); 
[10] L. R. Dodd, Phys. Rev. A 9, 637 (1974); Ian H. Sloan, Phys. Rev. A 7, 1016 (1973);

[11] C. Brezinski, "Pade - Type Approximation and General Orthogonal Polynomials," (Birkhauser, Basel, 1980); C. Brezinski and J. Van Iseghem, Pade approximation, in "Handbook of Numerical Analysis" (P. G. Ciarlet and J. L. Lions, Eds.), Vol. III, pp. 47 222, North-Holland, Amsterdam, 1994; R. Shakeshaft and X. Tang, Phys. Rev. A 35, 3945 (1987), R. Shakeshaft, ibid. A 37, 4488 (1988);

[12] G. Rawitscher and J. Liss, Am. J. of Phys., in press;

[13] S. Weinberg, Phys. Rev. 131, 440 (1963); ibid. 133 B 232 (1964); S. Weinberg and M. Scaldron, ibid. 133 B 1589 (1964); S. Weinberg in Lectures on Particles and Field Theory, Brandeis Summer Institute in Theoretical Physics (Prentice-Hall, Englewood Cliffs, 1964) Vol. 2, Chaps. 4 and 5;

[14] W. Glöckle and G. Rawitscher, Nucl. Phys. A 790, 282C (2007); W. Glöckle and G. Rawitscher, "Three-atom scattering via the Faddeev scheme in configuration space", physics/0512010 at arxiv.com; G. Rawitscher and W. Glöckle, EPJ Web of Conferences 3, 05012 (2010);

[15] E. O. Alt, P. Grassberger and W. Sandhas, Phys. Rev. D 1, 2581 (1970); C. Y. Chen and K.T. Chung, Phys. Rev A 2, 1449 (1970); P. J. Kramer and J. C. Y. Chen, Phys. Rev A 3, 568 (1971); W. Glöckle and R. Offerman, Phys. Rev. C 16, 2039 (1977);

[16] W. Glöckle, Private communication;

[17] R. Kosloff in Dynamics of Molecules and Chemical Reactions, edited by R.E. Wyatt and J. Z. H. Zhang (Marcel Dekker, New York, 1966); V. Kokoouline, O. Dulieu, R. Kosloff and F. Masnou-Seeuws, J. of Chem. Phys. 110, 9865 (1999);

[18] R. A. Gonzales, J. Eisert, I Koltracht, M. Neumann and G. Rawitscher, J. of Comput. Phys. 134, 134-149 (1997); R. A. Gonzales, S.-Y. Kang, I. Koltracht and G. Rawitscher, J. of Comput. Phys. 153, 160-202 (1999);

[19] A. Deloff, Ann. Phys. (NY) 322, 1373-1419 (2007); L. N. Trefethen, Spectral Methods in MATLAB, (SIAM, Philadelphia, PA, 2000) ; John P. Boyd, Chebyshev and Fourier Spectral Methods, 2nd revised ed. (Dover Publications, Mineola, NY, 2001); G. Rawitscher and I. Koltracht, Computing Sci. Eng. 7, 58 (2005); G. Rawitscher, Applications of a numerical spectral expansion method to problems in physics: A retrospective, in Operator Theory, Advances and Applications, Vol. 203, edited by Thomas Hempfling (Birkäuser Verlag, Basel, 2009), pp. 
409-426.

[20] G. Rawitscher and I. Koltracht, Eur. J. Phys. 27, 1179 (2006);

[21] G. Rawitscher, Phys. Rev. C 25, 2196 (1982);

[22] E. Zerrad, A. -S Khan, K. Zerrad, and G. Rawitscher, Can. J. Phys. 81: 1215 (2003);

[23] J. Stoer and R. Bulirsch, Introdution to Numerical Analysis (New York: Springer Verlag, 1980);

[24] G. Rawitscher, Iterative solution of integral equations on a basis of auxiliary Sturmian functions, submitted to Phys. Rev. A 